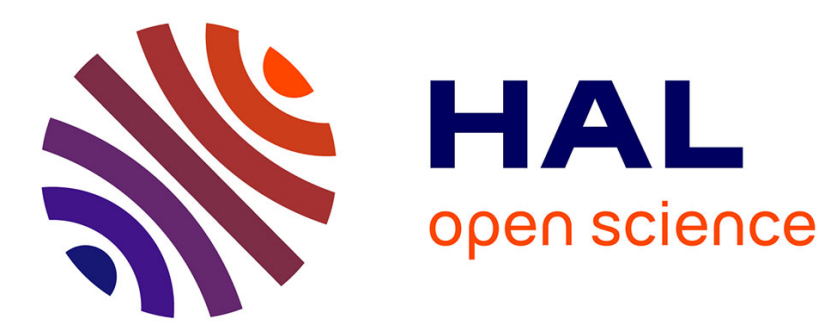

\title{
Isostatic deformations and polar wander induced by redistribution of mass within the Earth
}

\author{
Y. Ricard, R. Sabadini, G. Spada
}

\section{To cite this version:}

Y. Ricard, R. Sabadini, G. Spada. Isostatic deformations and polar wander induced by redistribution of mass within the Earth. Journal of Geophysical Research, 1992, 97 (B10), pp.14,223-14,236. 10.1029/92JB00740 . hal-02046596

\section{HAL Id: hal-02046596 https://hal.science/hal-02046596}

Submitted on 3 Feb 2021

HAL is a multi-disciplinary open access archive for the deposit and dissemination of scientific research documents, whether they are published or not. The documents may come from teaching and research institutions in France or abroad, or from public or private research centers.
L'archive ouverte pluridisciplinaire HAL, est destinée au dépôt et à la diffusion de documents scientifiques de niveau recherche, publiés ou non, émanant des établissements d'enseignement et de recherche français ou étrangers, des laboratoires publics ou privés. 


\title{
Isostatic Deformations and Polar Wander Induced by Redistribution of Mass Within the Earth
}

\author{
YANICK RICARD \\ Laboratoire de Géologie, Ecole Normale Supérieure, Paris, France \\ ROBERTO SABADINI ${ }^{1}$ AND GIORGIO SPADA ${ }^{2}$ \\ Dipartimento di Fisica, Settore Geofisica, Universita di Bologna, Bologna, Italy
}

\begin{abstract}
The effects at the surface of the Earth of time-dependent mantle mass anomalies are analyzed within the framework of a viscoelastic mantle with Maxwell rheology. The implications for the Earth's rotation are developed using the linearized liouville equations valid for small polar displacements. Our approach is appropriate for a simplified modeling of subduction. The displacement of the Earth's axis of rotation, called true polar wander, is very sensitive to the viscosity profile of the mantle and to the nature of the $670-\mathrm{km}$ seismic discontinuity. Phase change models generally yield a huge amount of polar wander, except for large viscosity increases. For chemically stratified models, true polar wander is drastically reduced as a consequence of dynamic compensation of the mass anomalies at the upper-lower mantle interface. When the source is embedded in the upper mantle in the proximity of the chemical density jump, the polar wander stops after a finite time controlled by the slowest isostatic mode.
\end{abstract}

\section{INTRODUCTION}

Up to now, the dynamics of the Earth's mantle submitted to loads has only been investigated for two simplified cases. On the one hand, the effects of time-independent internal loads on the resulting flow velocity, the induced dynamic topography, and the associated geoid have been studied [ $R \mathrm{i}$ card et al., 1984; Richards and Hager, 1984]. These models have been useful in understanding the mechanisms of compensation at long wavelengths. A satisfactory prediction of surface observations such as the geoid [Hager et al., 1985], the divergence of plate velocities [Forte and Peltier, 1987], or the plates rotation vectors [Ricard and Vigny, 1989] has been derived assuming that seismic tomography reveals the main density variations in the mantle. However, only steady state solutions have been computed. The transient time during which the dynamic topography is building up has not been taken into account except in very simple viscous models [Richards and Hager, 1984]. Furthermore, the effects on the rotation of the Earth of time-varying mass distributions within the mantle have never been considered.

On the other hand, the analysis of the Pleistocene deglaciation has focused on the effects of mass anomalies located at the surface of the planet [Cathles, 1975; Wu and Peltier, 1982]. The effect of cryospheric forcings on the rotational behavior of the Earth has also been treated by means of the linear version of Liouville equations with perturbations due to surface loads [Nakiboglu and Lambeck, 1980;

\footnotetext{
${ }^{1}$ Also at Istituto di Mineralogia, Università di Ferrara Ferrara, Italy.

${ }^{2}$ Now at Laboratoire de Géologie, Ecole Normale Supérieure, Paris, France.
}

Copyright 1992 by the American Geophysical Union.

Paper number 92JB00740.

0148-0227/92/92JB-00740\$05.00
Sabadini and Peltier, 1981; Sabarlini et al., 1984; Wu and Peltier, 1984]. These studies generated a new class of Earth models differing from the homogeneous viscoelastic sphere considered by Munk and MacDonald [1960], with more rea]istic mantle stratification. Some possible complexities, such as transient effects, were also added to the Maxwell rheology [Sabadini et al., 1985]. In these models, the mass anomalies forcing the displacement of the rotation axis and the variations in the length of the day are located at the surface of the planet. Some work has also been done with forcing terms located at the core-mantle interface [Hinderer et al., 1990; Lefftz and Legros, 1992].

We are thus in a situation of having a good insight into the dynamics associated with forcing terms acting at the top or at the base of the mantle. However, we know very little about the time variability of the isostatic and rotational responses of the planet to perturbing densities embedded in the mantle. As a consequence of this, several papers have been published in which the effects of internal mass anomalies have only been considered for a rigid Earth [Jurdy, 1983; Tanimoto, 1989], without allowing for the dynamics of a fluidlike mantle. The importance of dynamic compensation on the moment of inertia associated with the internal mass anomalies inferred from seismic tomography has recently been addressed by Ricard and Sabadini [1990] for the case of a viscous mantle.

The pole excursions, called true polar wander (TPW), have been detected by paleomagnetic studies for the last 200 Ma [Hargraves and Duncan, 1973; Besse and Courtillot, 1991]. This TPW is deduced by comparison of two kinds of trajectories: the apparent polar wander paths (APWP) and the hotspot tracks. The APWP of a craton characterizes the motion of a stable lithosphere with respect to the main magnetic field which is assumed to remain parallel to the Earth's rotation axis. The trajectory of the same craton with respect to the hotspots defines the relative motion between lithosphere and mantle [Morgan, 1971].

As has been suggested by different authors, the changes in surface plate tectonics and the creation of new subduc- 
tion trenches modify the Earth's inertial axis and are probably the main driving phenomena of long-term polar wandering [Goldreich and Toomre, 1969; Courtillot and Besse, 1987; Duncan and Richards, 1991]. It is thus of prime importance to understand the quantitative relationships between the time variations of internal mass distributions and the induced changes in the Earth's rotation. Careful reconstructions of the geographic distribution of the subduction pattern and paleomagnetic data should be used in dynamic models of the Earth in order to constrain the mechanical properties of the mantle.

In this paper we show the effects of viscoelastic mantle stratification and of moving mass anomalies appropriate for the modeling of lateral density variations caused by subduction on the Earth's rotation. We focus in particular on the effects linked with a viscosity increase in the lower mantle and a chemical stratification at the $670-\mathrm{km}$ discontinuity. The results of our model are restricted to small polar displacements, as we solve a linearized version of the Liouville equation.

\section{VISCOELASTIC COMPENSATION oF INTERNAI, SOURCES}

Making use of the correspondence principle, viscoelastic problems can be treated as elastic problems in the Laplace domain. A given mass anomaly embedded in the mantle induces an elastic response and a delayed viscoelastic flow that perturb the various interfaces of the Earth. The induced perturbation of the gravity potential expressed by a spherical harmonic of degree $l, \delta V_{l}$ is usually written in the following form:

$$
\delta V_{l}(s)=\left[1+k_{l}(s)\right] \delta V_{l}^{0}(s),
$$

where $k_{l}$ is the internal loading potential Love number [Kaula, 1963; Hinderer et al., 1991]. The term $\delta V_{l}^{0}(s)$ represents the direct effect of internal masses on the potential. Its time variations are taken into account through the Laplace variable $s$. This term would be the only contribution to the potential in a rigid Earth. However, in a viscoelastic Earth, the deflection of the interfaces induces an additional effect. which tends to cancel the direct effect of internal masses. This compensation is taken into account by the term $k_{l} \delta V_{l}^{0}$.

The Love number $k_{l}$ depends on the mechanical stratifcation of the model Earth and is a function of the Laplace variable $s$. In a very general way it can be written as the sum of an elastic contribution $k_{l}^{e}$ plus a sequence of terms associated with the viscoelastic relaxation modes

$$
k_{l}=k_{l}^{e}+\sum_{i=1}^{M} \frac{k_{l}^{i}}{s-s_{i}}
$$

For an impulse excitation of the Earth, equation (2) indicates that the Love number is an elastic impulse plus the sum of $M$ contributions of amplitudes $k_{l}^{i}$ decaying exponentially with characteristic times $-1 / s_{i}$. For a more complex excitation, a convolution must be performed between the temporal evolution of the load and the $M$ modes. The number $M$ depends on the density and viscosity stratification of the Earth. The characteristic relaxation times $-1 / s_{i}$ are deduced from the resolution of a secular equation that only depends on the mechanical properties of the Earth. These times are the usual relaxation times entering postglacial rebound problems. The coefficients $k_{i}^{e}$ and $k_{l}^{i}$ are derived from the solution of an appropriate boundary value problem. They depend both on the Earth's mechanical structure and on the location of the load in the mantle. This paper thus generalizes the usual case of postglacial rebound processes where the $k_{l}^{e}$ and $k_{l}^{i}$ are only computed for surface loads.

In what follows, we make use of different Earth models consisting of an inner inviscid core, an upper and lower mantle with Maxwell rheology separated by a phase change, or a chemical boundary at $670 \mathrm{~km}$. An elastic lithosphere is present in some of our models. The number of modes varies from $M=2$ to $M=6$ depending on the nature of the 670-km depth interface and on the presence of a lithosphere [Yuen et al., 1982]. In Table 1 the parameters of eight different rheological models, from a to $h$, are listed. In the remainder of this paper, the letters a to $h$ labeling the different figure panels will refer to the rheological model having the same letter in Table 1. The different relaxation modes consist of two transient modes (T1 and T2), a lithospheric mode (L0), and two modes where the Earth surface and the core-mantle boundary are moving in the same direction (mantle mode M0) or in the opposite direction (core mode $\mathrm{C} 0$ ), respectively. A slow mode (M1) is associated with the deformation of the flow barrier at the upper-lower mantle interface in models $\mathrm{e}, \mathrm{f}, \mathrm{g}$, and $\mathrm{h}$. This mode disappears when

\begin{tabular}{|c|c|c|c|c|c|c|c|c|c|}
\hline Model & ${ }_{\mathbf{k g} \mathbf{m}^{-3}}^{\rho_{L}}$ & $\begin{array}{c}\rho_{1} \\
\mathbf{k g ~ m}^{-3}\end{array}$ & $\begin{array}{c}\rho_{2}, \\
\mathbf{k g ~ m}^{-3}\end{array}$ & ${ }_{\mathrm{kg} \mathrm{m}}^{\rho_{C}}$ & $\underset{\mathrm{Pa} \times 10^{11}}{\mu L}$ & $\underset{\mathrm{Pa}_{\mathrm{a}} \boldsymbol{\mu}_{1}}{\mu^{11}}$ & $\begin{array}{c}\mu_{2} \\
\mathrm{~Pa} \times 10^{11}\end{array}$ & $\begin{array}{c}\nu_{1} \\
\mathrm{~Pa} \times 10^{21}\end{array}$ & $\begin{array}{c}\nu_{2}, \\
\operatorname{Pa} \times 10^{21}\end{array}$ \\
\hline $\mathbf{a}$ & $\ldots$ & 4314 & 4314 & 10926 & $\ldots$ & 1.45 & 1.45 & 1 & $\mathbf{1}$ \\
\hline $\mathbf{b}$ & 4314 & 4314 & 4314 & 10926 & 1.45 & 1.45 & 1.45 & 1 & 1 \\
\hline c & $\ldots$ & 4314 & 4314 & 10926 & $\ldots$ & 1.45 & 1.45 & 1 & 30 \\
\hline d & 4314 & 4314 & 4314 & 10926 & 1.45 & 1.45 & 1.45 & 1 & 30 \\
\hline e & $\ldots$ & 4120 & 4508 & 10926 & $\ldots$ & 1.38 & 1.51 & 1 & 1 \\
\hline f & 4120 & 4120 & 4508 & 10926 & 1.38 & 1.38 & 1.51 & 1 & 1 \\
\hline g & $\ldots$ & 4120 & 4508 & 10926 & $\ldots$ & 1.38 & 1.51 & 1 & 30 \\
\hline $\mathbf{0}$ & 4120 & 4120 & 4508 & 10926 & 1.38 & 1.38 & 1.51 & 1 & 30 \\
\hline
\end{tabular}

TABLE 1. Physical Properties of the Eight Earth Models

Symbols $\rho, \mu$, and $\nu$ indicate density, elastic rigidity, and viscosity, respectively. Labels $L, 1$, and 2 refer to lithosphere, upper mantle, and lower mantle, respectively. 
TABLE 2. Relaxation Times of the Viscoelastic Modes

$\begin{array}{ccccccc} & \text { T1, } & \text { T2, } & \text { Lo, } & \text { M0, } & \text { Co, } & \text { M1, } \\ \text { Model } & \text { years } & \text { years } & \text { years } & \text { years } & \text { years } & \text { years }\end{array}$

\begin{tabular}{|c|c|c|c|c|c|c|}
\hline $\begin{array}{l}\mathbf{a} \\
\mathbf{b} \\
\mathbf{c} \\
\mathbf{d} \\
\mathbf{e} \\
\mathbf{f} \\
\mathbf{g} \\
\mathbf{h}\end{array}$ & $\begin{array}{c}\cdots \\
2.36 \times 10^{2} \\
2.34 \times 10^{2} \\
2.21 \times 10^{2} \\
2.22 \times 10^{2} \\
2.46 \times 10^{2} \\
2.43 \times 10^{2}\end{array}$ & $\begin{array}{c}\cdots \\
3.25 \times 10^{2} \\
3.07 \times 10^{2} \\
2.26 \times 10^{2} \\
2.27 \times 10^{2} \\
3.31 \times 10^{2} \\
3.13 \times 10^{2}\end{array}$ & $\begin{array}{c}4.74 \times 10^{2} \\
\cdots \\
6.96 \times 10^{3} \\
\cdots \\
4.64 \times 10^{2} \\
\cdots \\
7.23 \times 10^{3}\end{array}$ & $\begin{array}{l}4.92 \times 10^{2} \\
1.88 \times 10^{3} \\
1.28 \times 10^{4} \\
1.28 \times 10^{4} \\
4.81 \times 10^{2} \\
1.91 \times 10^{3} \\
1.24 \times 10^{4} \\
1.24 \times 10^{4}\end{array}$ & $\begin{array}{l}2.53 \times 10^{3} \\
6.16 \times 10^{3} \\
4.80 \times 10^{4} \\
7.59 \times 10^{4} \\
2.54 \times 10^{3} \\
6.18 \times 10^{3} \\
4.83 \times 10^{4} \\
7.86 \times 10^{4}\end{array}$ & $\begin{array}{c}\cdots \\
\cdots \\
\cdots \\
2.40 \times 10^{5} \\
1.58 \times 10^{6} \\
1.13 \times 10^{6} \\
4.25 \times 10^{6}\end{array}$ \\
\hline
\end{tabular}
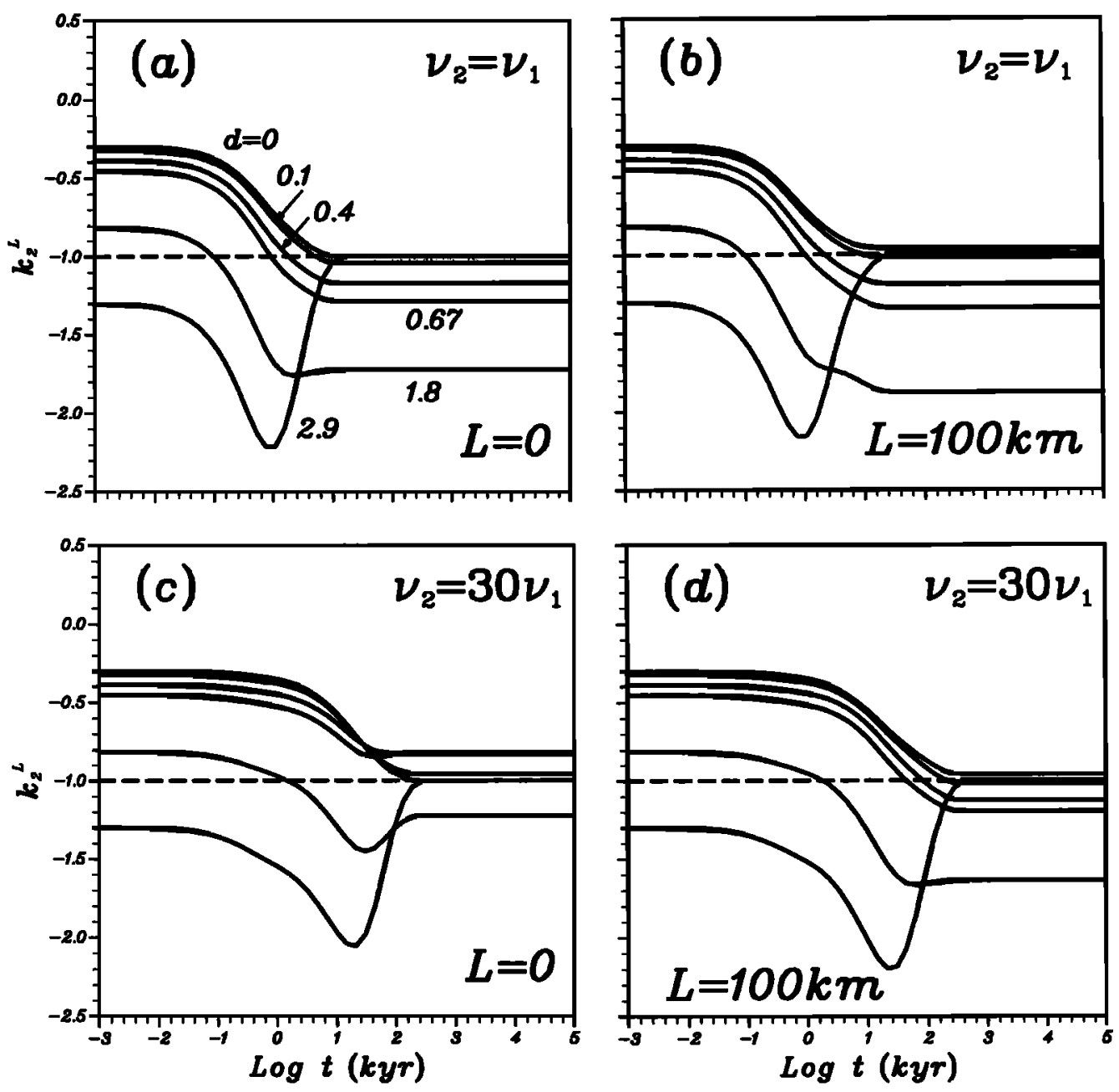

Fig. 1. Time evolution of the loading Love number $k_{2}$ for different mantle stratifications. The mass anomalies are located at six depths sampling the whole mantle. These depths are given in units of $1000 \mathrm{~km}$ in the top left panel for model a. In the top row the mantle is uniform. The lower mantle is $\mathbf{3 0}$ times more viscous than the upper mantle in the bottom row. In the left column a free slip boundary condition is imposed at the Earth's surface whereas in the right column a purely elastic lithosphere of $100 \mathrm{~km}$ thick surrounds the mantle. An adiabatic phase change is assumed at the lower-upper mantle interface. A Love number greater than -1 indicates than the gravity potential has the sign of the exciting deep mass anomaly. On the contrary, a Love number less than -1 , which is generally the case, means that the gravity contributions associated with the induced dynamic topographies overcome the direct effect of the deep mass. 
a phase change boundary condition is imposed at $\mathbf{6 7 0 - \mathrm { km }}$ depth. In some of our computations the lithosphere is not included (models a, c, e, and $g$ ) and the number of modes is reduced by the absence of the L0 mode. The two transient modes are associated with a small $k_{i}^{i}$. Their influence on the behavior of the Earth is negligible.

The computation of the characteristic times $-1 / s_{i}$ is now a classical problem [Sabadini et al. 1982; Wu and Peltier, 1982]. For each of our eight Earth models, the relaxation times expressed in years are listed in Table 2. To solve the appropriate boundary value problem and derive the $k_{l}^{e}$ and $k_{l}^{i}$ factors, we used the explicit expression of the inverse of the fundamental matrix which propagates the solution in each layer [Spada et al., 1990]. We mainly emphasize the effect of upper mantle sources as we want to focus on the relationship between the polar motion and an evolving subduction zone.

The time evolutions of the loading Love number $\boldsymbol{k}_{l}$ for degree 2 and for different depths of the source are portrayed in Figures 1 and 2. These time evolutions have been computed assuming that a positive density anomaly starts to act at time $t=0$ and remains constant thereafter. Different. possible mantle viscoelastic profiles have been used. A Love number greater than -1 indicates that the gravity perturbation has the sign of the mass anomaly. On the contrary, a Love number less than -1 means that the effects of the surface deformation dominate. Perfect compensation for the gravity potential would require that the asymptotic value of $k_{l}$ attains the value -1 , in order to cancel the term +1 entering equation (1).

In Figure 1, a phase change condition is applied at the upper-lower mantle boundary. The upper mantle viscosity is $\nu_{1}=10^{21} \mathrm{~Pa} \mathrm{~s}$. The mantle is uniform in the top row (a and b) whereas the lower mantle viscosity $\nu_{2}$ has been increased to $\nu_{2}=30 \nu_{1}$ in the bottom row (c and d). No lithosphere has been added in the computations of the left column ( $a$ and $c$ ), whereas an elastic lithosphere of thickness $L=100 \mathrm{~km}$ is present in the right column (b and d). The absence of an elastic lithosphere corresponds to a free-slip boundary condition on the top of the planet. In addition to the two modes ( $\mathrm{MO}$ and $\mathrm{CO}$ ), the third mode ( $\mathrm{L} 0$ ) is acting in the right column. The mass anomalies are embedded at six different depths $(0,100,400,670,1800,2900 \mathrm{~km})$ spanning the whole mantle.
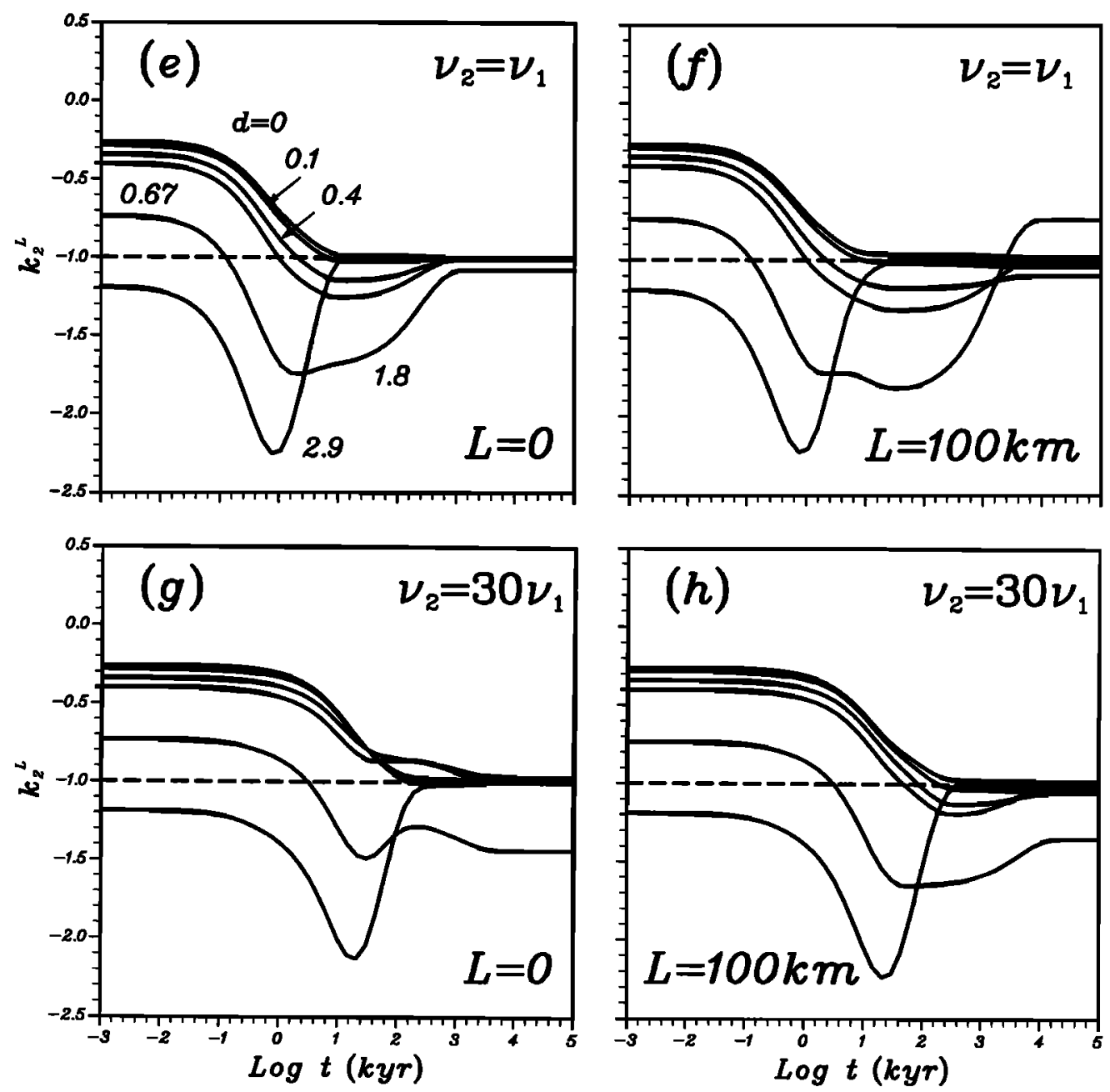

Fig. 2. Same as in Figure 1 but for Earth models with a nonadiabatic stratification at the 670-km depth interface. Owing to the action of the slow M1 mode, the Love numbers reach their asymptotir values after a much longer time than for the phase transition models. After a few million years the Love numbers are greater or less than minus 1 depending on the mantle viscosity stratification. 
For all depths, an asymptotic value for the Love number is reached after a few 1000 years, i.e., after a time corresponding to the usual characteristic time of postglacial uplift [Haskell, 1935]. For a uniform mantle without lithosphere (model a of Figure 1), the asymptotic values are always less than -1 . Therefore, the sign of the geoid induced by a given mass is opposite to the sign of the mass itself. The existence of a lithosphere on top of a uniform mantle (b), makes the Love numbers for internal loads even more negative. These two remarks have already been stated in the framework of steady state models [Ricard et al., 1984; Richards and Hager, 1984]. The physical explanation is simple: the topography induced by the viscoelastic flow, being closer to the observer than the deep-seated mass, imposes its sign to the gravity field. The existence of the lithosphere concentrates the stresses above the mass anomaly and leads to larger deflections. In the upper right panel (model b) it can be seen that a mass anomaly seated on the lithosphere is partially sustained by elastic stresses and is associated with a Love number slightly larger than -1 even in the asymptotic regime.

In models $c$ and $d$ of Figure 1, because of the larger average viscosity of the model Earth, the asymptotic regime is reached after a somewhat larger time than in the first row. As was already observed in steady state computations, the Love number evolves to values that are less or greater than -1 , depending on the position of the mass with respect to the depth of the viscosity increase and depending on the presence or not of a lithosphere. Here again the explanation comes from the magnitude of the induced surface deformation. The stiffer lower mantle is able to sustain more stresses; as a consequence, the surface topography is of smaller amplitude and the Love number is closer to -1 .

The effects linked to a chemical transition at $670 \mathrm{~km}$ on the time evolution of the loading Love number are depicted in Figure 2. As in Figure 1, the top row (e and $f$ ) is for an isoviscous mantle, and the bottom row ( $g$ and $h$ ) is for a stiffer lower mantle $\left(\nu_{2}=30 \nu_{1}\right)$. The upper mantle viscosity remains fixed at $\nu_{1}=10^{21} \mathrm{~Pa}$ s. The mass anomalies are located at the same depths as in Figure 1. In the left panels (models $\mathrm{e}$ and $\mathrm{g}$ ) there is no lithosphere, while a 100-kmthick elastic lithosphere is introduced in the model used for the right panels (models $f$ and $h$ ). There is a new feature that is brought out by the chemical stratification: the compensation of the density anomaly when the source is moved close to the transition depth. On time scales of the order of one million years the extra viscoelastic relaxation mode $M 1$, connected to the density jump at $670 \mathrm{~km}$, begins to play a role, allowing for the isostatic compensation of the density anomaly. The steady state limit is reached after a time increasing with the magnitude of the lower mantle viscosity and decreasing in the presence of an elastic lithosphere. A mass anomaly located close to a chemical interface, being locally compensated by the deflection of this interface, leads to a Love number equal to -1 in the asymptotic regime.

The results depicted in Figures 1 and 2 are obtained for a reference viscosity in the upper mantle of $\nu_{1}=10^{21} \mathrm{~Pa} \mathrm{~s}$. The temporal scale of the compensation process is simply proportional to this reference viscosity. This statement would be strictly valid for a purely viscous mantle but remains correct at first order for our viscoelastic model.

Another way to look at the time-dependent evolution of the isostatic compensation is to consider the changes in the geoid as a function of time. In Figure 3, masses of degree 2 located at different depths are starting to act at time $t=$ 0. The Green's function relating these masses to the geoid are plotted for several times after the loading. The eight panels (models a to $h$ ) correspond to the eight panels of Love numbers depicted in Figures 1 (models $a$ to $d$ ) and 2 (models e to h). At $t=0$, just after the elastic shot, the deep mass remains strongly undercompensated, and the gravity potential is mainly related to the mass itself. In that case, the gravity potential roughly decreases as the power 4 of the radius at which the mass is located [e.g., Lambeck, 1988]. After a few thousand years the asymptotic regime $(t=\infty)$ is attained, and for mass anomalies located close to the surface, the geoid height is simply proportional to the compensation depth [Ockenden and Turcotte, 1977]. In the asymptotic regime, our solutions depicted by thick lines reach the values obtained with steady state computations [Ricard et al., 1984; Richards and Hager, 1984]. We observe that the viscosity profile within the Earth and the existence or absence of a lithosphere determine the sign of the geoid.

The previous computations show the limits of the steady state models. The Earth's surface only relaxes after times that can be of the order of 10 million years if the mantle is strongly stratified. Assuming velocities in the mantle of the order of $10 \mathrm{~cm} / \mathrm{yr}$, a transport of about $1000 \mathrm{~km}$ can occur before the Earth's surface reaches its final deflection. This transport length remains much smaller than the wavelength of the anomalies of degree 2 that we have considered but cannot be totally neglected.

For simple models of postglacial rebound in Cartesian coordinates the characteristic relaxation time of a homogeneous half-space is inversely proportional to the wavelength of the surface perturbation [Haskell, 1935]. This suggests that for shorter-wavelength anomalies the characteristic time of surface deflection can be comparable to the characteristic time of mantle convection. Therefore, we compute the evolution of the Earth's relaxation for a load expressed by a spherical harmonic of degree $l=16$. Figure 4 depicts for this degree the geoid Green's function for different times and different mantle viscosity profiles in the same manner as in Figure 3. The slowing down of characteristic times with wavenumber that is expected from Cartesian geometry and uniform mantle is not observed in our spherically stratified model. In the right panels the asymptotic regime is reached after comparable times for degree 2 and 16: this agrees with the behavior of the different relaxation modes with degree. Of course, for higher degrees, the behavior of our model approches the results obtained with a Cartesian geometry. Among the different isostatic modes, only the M0 mode has a characteristic time increasing with $l$ for low degrees [Spada et al., 1992; $W u, 1990]$. This explains why only in the left top panel, where the L0 and M1 modes are absent, the solutions for $l=16$ evolve more slowly toward the asymptotic regime than for the case $l=2$.

Our results suggest that the modeling of geoid anomalies based on the viscous limit of the response functions, as it has been done until now, is only correct within the assumption that the density pattern does not change on time scales of million of years; this may not be correct for fast thermal instabilities with short wavelength [Yuen and Fleitout, 1985; Fleitout and Moriceau, 1991]. 

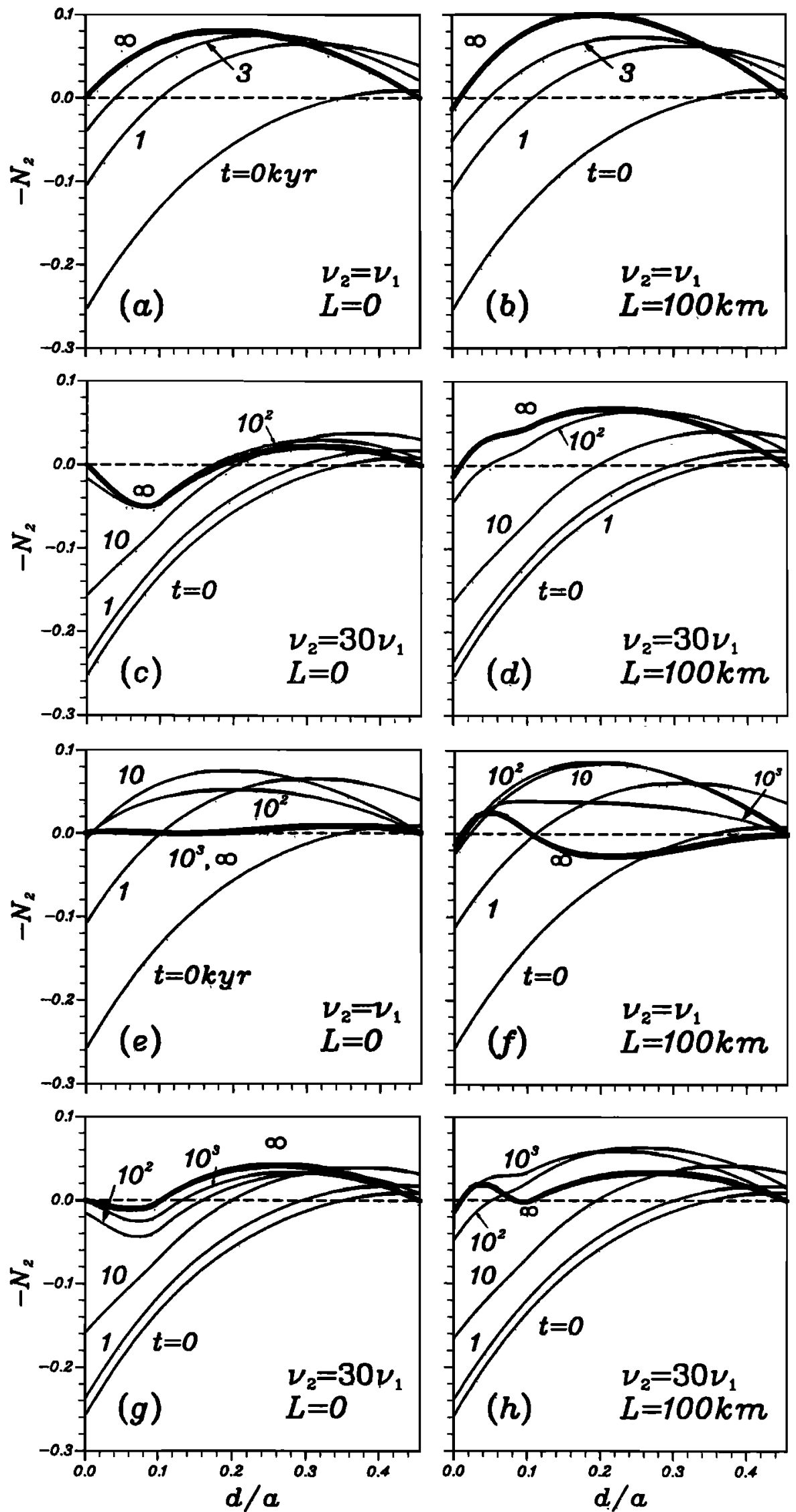

Fig. 3. Geoid perturbations induced by a mass anomaly of degree 2 located in the mantle. The depth of the mass anomaly $d$, normalized by the Earth's radius a, ranges from the surface to the core-mantle boundary. The perturbations have been computed at different times $t$ expressed in kyloyears after the beginning of loading. The thick line of each panel provides the steady state solutions $(t=\infty)$. The top four panels (models a to $d$ ) have the same mantle stratifications as in Figure 1 where a phase change is assumed at the lower-upper mantle interface. The left column corresponds to models without lithosphere and the right column corresponds to models with lithosphere. The mantle is of constant viscosity in models a and $b$, and the lower mantle viscosity is increased by a factor of 30 in models $c$ and $d$. The same mantle stratifications but assuming a chemical boundary at 670-km depth (see Figure 2) are considered in the four bottom panels (models e to h). 

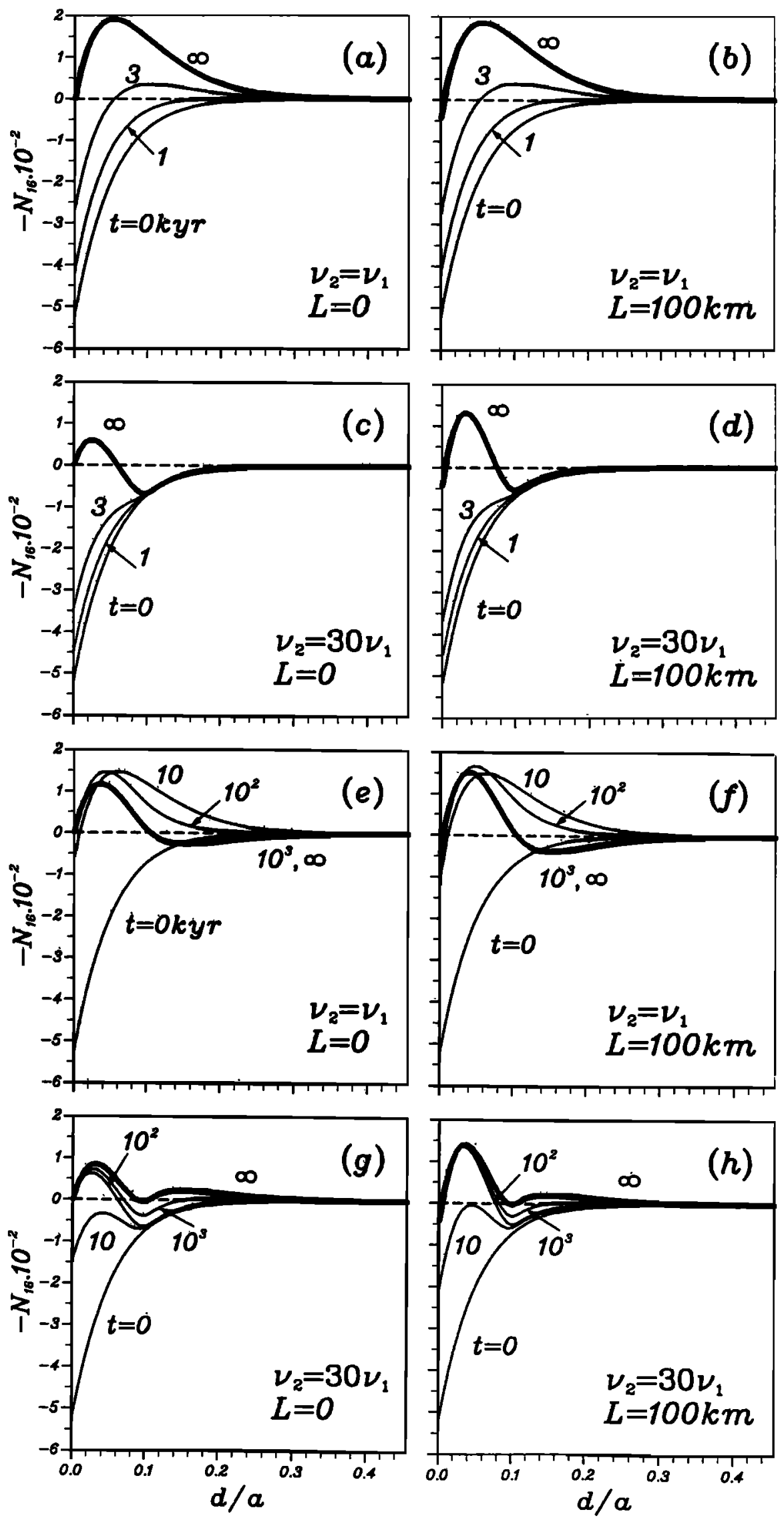

Fig. 4. Geoid perturbations induced by a mass anomaly of clegree $l=16$ located in the mantle. Except for the loading degree, this figure is similar to Figure 3. 


\section{ROTATIONAL DYNAMICS FOR INTERNAL SOURCES}

The excursions of the Earth's axis of rotation with respect to the geography (TPW) can be described by the linearized Liouville equation that takes the following form in the Laplace domain [Sabadini and Peltier, 1981]

$$
\left(\frac{i}{\sigma_{r}} s+1\right) \mathbf{m}(s)=\psi(s)+\phi(s),
$$

where $m=m_{1}+i m_{2}$ is a complex variable built from the two director cosines $m_{1}$ and $m_{2}$ of the axis of rotation in the geographical coordinate frame, and $\sigma_{r}$ is the Chandler wobble frequency. Equation (3) neglects the possible differential rotation of the fluid core with respect to the mantle [Hinderer et al., 1982]. This effect is of very small importance for our study.

The term in $\psi(s)$ includes the changes in the off-diagonal terms of the inertial tensor $I_{x z}$ and $I_{y z}$. It takes into account both the effect of internal masses and the effect of the induced interface deflections that simultaneously perturb the inertial tensor of the nonrotating Earth. It can easily be shown that the inertial tensor is related to the nonhydrostatic geoid anomalies of degree 2 only. In particular, $I_{x z}$ and $I_{y z}$ are proportional to the potential anomalies of degree 2 and order 1 . Therefore, only these components of the perturbing masses enter in this forcing term. The $\phi(s)$ excitation is the additional contribution coming from the changes in the centrifugal potential occurring during polar wander. Its physical origin is very simple: because of its rotation the Earth has an equatorial bulge, and any change of its rotation axis gives rise to a new stabilizing contribution to the nondiagonal inertial terms. The $\phi(s)$ term characterizes thus the ability of the planet to readjust its equatorial bulge by viscous yielding. Its contribution is proportional to the temporal changes in $\mathrm{m}(s)$ and therefore proportional to $s \mathbf{m}(s)$ in the Laplace domain. For a stratified viscoelastic Earth with Maxwell rheology, its exact form is again a sum of isostatic modes but derived from a tidal boundary problem of degree 2 instead of a loading problem [Takeuchi et al., 1962; Sabadini et al., 1982]. The tidal problem of degree 2 and the rotational problem are similar, as they both involve formally the same volume potential.

We can gain some insight into the physics of the rotational readjustment of the planet from the analysis of viscoelastic relaxation modes excited by a tidal loading. We saw that the characteristic times entering in loading problems with internal masses or surface loads are the same, only the amplitudes $k_{l}^{e}$ and $k_{l}^{i}$ are different. Here again the same characteristic times $t_{i}=-1 / s_{i}$ appear but are associated with tidally excited residues. Let us consider a model Earth which presents a chemical boundary at $670-\mathrm{km}$ depth and carries six modes. In Figure 5 the stream functions associated with the deformation of the various modes (model $h$ ) are depicted in a polar cross section of the northern hemisphere of the Earth. The azimuth of the cross section is perpendicular to the zero meridian of the degree 2 and order 1 spherical harmonic component. The level lines of a stream function are, by definition. parallel to the velocity vectors. The excitation being of degree 2 and order 1 , the velocity is purely radial at $45^{\circ}$, and the level lines in the southern hemisphere can be deduced by symmetry. The sign of the radial velocity changes from the left side to the right side of each panel, so that the spherical Earth tends to flatten
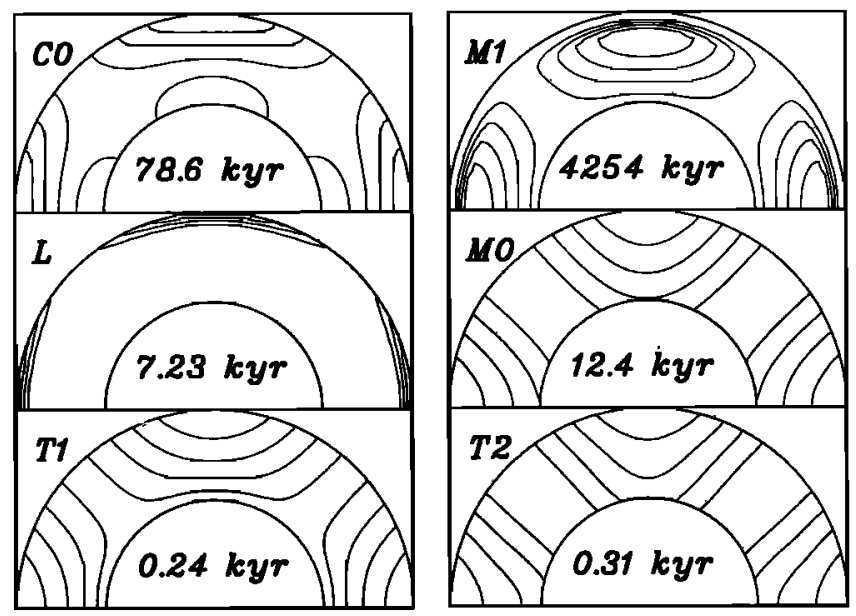

Fig. 5. Viscoelastic relaxation modes (model h) excited by a tidal load of degree 2 and order 1 . The stream function of the deformation is depicted in a polar cross section of the northern hemisphere of the Earth. The names of the modes and their characteristic times are written in each panel. Except for the M1 mode, all the other modes tend to deform the Earth surface and to change the spherical shape of the model Earth into an ellipsoidal shape inclined at $15^{\circ}$.

around an axis at $45^{\circ}$. The results are representative of the eigen-deformation of the planet induced by variations in the centrifugal potential during polar wander. In the real wandering Earth, the circulation depicted in this figure must be added to the flow driven by the mass anomalies and to the slow drift of the geographical coordinates in the inertial frame.

The relaxation modes are ordered by increasing characteristic times. The bottom panels correspond to very fast decaying modes (T1 and T2). The lithospheric mode (L0) can be easily recognized in the left panel of the middle row. The core $(\mathrm{C} 0)$ and mantle (M0) modes correspond to symmetrical and antisymmetrical deformations, respectively, at the surface and at the core mantle boundary. These last three modes have characteristic times comparable to the age of the last deglaciation. The slowest mantle mode (M1), due to the chemical stratification, is portrayed in the right panel of the top row. Its characteristic time is more akin to the characteristic times of mantle convection. This mode is the only one having closed level lines, i.e., a zero vertical velocity on both sides of the mantle. The contribution of this mode has proven to be crucial in our calculations, and from the deformation pattern we can see the large shear induced in the upper mantle during the readjustment of the rotational bulge, with flow essentially concentrated in the outer portion of the planet.

Three temporal scales are interacting in equation (3): the intrinsic time variation of internal masses, the time variation of the isostatic process excited by loading, and finally the time variation of the isostatic process excited by rotational changes. The dynamics of rotation is thus much more complex than the simple loading problem. However, the solution of the loading problem of degree 2 is the necessary first step toward the resolution of the Liouville equations.

Equation 3 can be rewritten by moving the $\phi(s)$ excitation in the left-hand member and grouping all the terms containing $\mathbf{m}(s)$ on the form of a rational fraction of two 
polynomials in $s$ [Sabadini et al., 1984]. After expressing this function of $s$ in monomial factors, equation (3) reads:

$$
\mathbf{m}(s)=\left(\frac{\mathbf{A}_{1}}{s}+\sum_{i=1}^{M} \frac{\mathbf{A}_{i+1}}{s-\mathbf{a}_{i}}\right) \frac{I_{x z}(s)+i I_{y z}(s)}{C-A},
$$

where $C-A$, the difference between the polar and equatorial inertia of the Earth, characterizes the equatorial bulge.

The term $I_{x z}(s)+i I_{y z}(s)$ denotes the perturbation in the off-diagonal components of the inertia tensor due to the internal density anomalies and induced flow in a nonrotating mantle. In the real rotating Earth, these terms are balanced by the readjustement of the equatorial bulge in such a way that the angular distance between the axis of rotation and the main inertial axis is just the amplitude of the Chandler wobble (presently about $10 \mathrm{~m}$ ). In other words, the inertial perturbations entering equation (4) are only related to the $\psi(s)$ terms, whereas the effective inertial perturbations of the rotating Earth are related to $\psi(s)+\phi(s)$.

Following equation (2), the contribution of the offdiagonal inertia tensor can easily be quantified if we introduce the Love number $k_{2}(s)$ of degree 2 :

$$
\frac{I_{x z}(s)+i I_{y z}(s)}{C-A}=\left[1+k_{2}(s)\right] f(s) .
$$

In equation (5), the function $f(s)$ denotes the time dependence of the degree 2 and order 1 components of potential induced by the exciting density anomaly. Of course, a punctual mass anomaly located at the equator or at the poles has no such components and cannot drive any polar wander.

The inverse Laplace transform of the part of equation (4) that includes the $\mathbf{A}_{\mathbf{i}}$ and $\mathbf{a}_{\mathbf{i}}$ terms leads to a temporal function consisting in a linear term and a sum of exponentially decaying terms with characteristic times $-1 / \mathbf{a}_{\mathbf{i}}$. The complex residue $\mathbf{A}_{1}$ accounts for the steady state polar wander, and it generalizes, for a stratified, viscoelastic Earth, the secular term used by Munk and MacDonald [1960] to estimate the amount of polar drift driven by the distribution of the continents. The set of complex residues $\mathbf{A}_{i+1}$ and inverse complex relaxation times $a_{i}$ describes the transient contributions. To each of the isostatic characteristic times, $-1 / s_{i}$ corresponds a rotational time $-1 / \mathbf{a}_{\mathbf{j}}$. Numerically, all these times appear to be real except one that carries the Chandler wobble in its imaginary part and is associated with the Mo mode [Sabadini et al., 1984]. In what follows, the Laplace transform of (4) will be performed after having filtered out this imaginary component. Its physical meaning and characteristic period are indeed not related to true polar wander. The different rotational modes have generally been called $\mathrm{M} 1, \mathrm{~L} 0, \mathrm{M} 0, \mathrm{C} 0, \mathrm{~T} 1$, or $\mathrm{T} 2$ according to the isostatic mode having a comparable time constant. However, contrary to the isostatic modes, they do not correspond to a given flow pattern and their names are rather arbitrary.

In Figure 6 the characteristic rotational and isostatic times are plotted as a function of the ratio between the lower and upper mantle viscosities for Earth models with an adiabatic phase change at $670-\mathrm{km}$ depth (top) or with a chemical boundary (bottom). The lithosphere is absent in the left column. The transient $\mathrm{T}$ modes are not plotted. The M1 mode is absent in the top row, and the Lo mode only acts in the right column. The rotational modes are generally faster than their isostatic corresponding modes except for the mantle mode M0. This means that the rotational behavior is rapidly controlled by the secular term $\mathbf{A}_{1}$.

The secular term $\mathbf{A}_{1}$ controls the constant velocity of polar wander reached after a time larger than the isostatic and rotational times. This term depends on the stratification of the Earth. A viscosity increase at $670 \mathrm{~km}$-depth slows down the polar wander, particularly when a chemical boundary is also present [Sabadini and Yuen, 1989; Ricard and Sabadini, 1990]. For a viscosity increase larger than 1 order of magnitude, the effects of chemical and phase change stratifications are basically indistinguishable.

\section{Polar Wander INDUCED by SUBduction}

We can now solve equation (4) for a given temporal and spatial dependence of a mass anomaly. We first choose a mass anomaly that mimics a slab located at $45^{\circ}$ from the north pole. This slab is supposed to be punctual and located at a given depth in the mantle. Its mass corresponds to a slab pull of $5 . \times 10^{13} \mathrm{~N} / \mathrm{m}$ [Turcotte and Shubert, 1982] over a trench length of $4000 \mathrm{~km}$. The slab acts suddenly at time $t=0$. Its location at latitude $45^{\circ}$, provides the maximum effect on polar displacement. A slab acting at a different latitude $\theta$ would induce an initial polar drift velocity reduced by the factor $0.5 \sin 2 \theta$.

The mass of our slab is around $2.0 \times 10^{19} \mathrm{~kg}$, a value which is close to the ice sheet masses that covered the Laurentides $\left(1.8 \times 10^{19} \mathrm{~kg}\right)$, Fennoscandia $\left(6.0 \times 10^{18} \mathrm{~kg}\right)$, and Antarctica $\left(1.6 \times 10^{19} \mathrm{~kg}\right)$ before the Pleistocenic deglaciation. According to different authors [Sabadini and Peltier, 1981; Wu and Peltier, 1984], the disintegration of these ice sheets is driving the actual observed polar wander of about $1 . \% \mathrm{~m} . \mathrm{y}$. [Dickman, 1981]. Of course, only the load contribution on the degree 2 and order 1 Legendre polynomial enters in the amplitude of the driving function $f(s)$ of equation (5). This polynomial being a function of the sine of two times the colatitude, the Antarctic ice sheet centered at the south pole did not drive any polar wander. The quasi-polar location of the Laurentide and Fennoscandia ice sheets, at $25^{\circ}$ of the north pole, affected the rotation of the Earth by only $76 \%$ of the effect of our model slab located at $45^{\circ}$

In solving equation (4) we make the approximation that the source mass always stays at constant latitude. In a more realistic Earth model, the source should be moved according to the polar wander itself. However, as long as the amplitude of the polar displacement is less than about $10^{\circ}$, the amplitude of the mass component of degree 2 and order 1 is not too strongly affected by the change in latitude. For simplicity, in these first step computations, we make the hypothesis of a fixed source, but the reader should be aware that our approximation breaks down as soon as the computed polar wander is larger than $10^{\circ}$ or $15^{\circ}$. It is also obvious that the polar wander excited by our mass located at $45^{\circ}$ at $t=0$ cannot be extrapolated to pole displacements larger than $45^{\circ}$. As was explained before, we also filter out the Chandler wobble by equating to zero the imaginary part of the rotational time associated with the mantle Mo mode. The real trajectories of the pole are in fact spiraling at the Chandler period (14 months) with a decay time of a few tens of Chandler periods [W'ilson and Vicente, 1981] around the trajectories we computed.

In Figure 7, we depict the displacements of the axis of rotation induced by our mass anomaly with respect to the geographic reference frame. This anomaly is located at dif- 

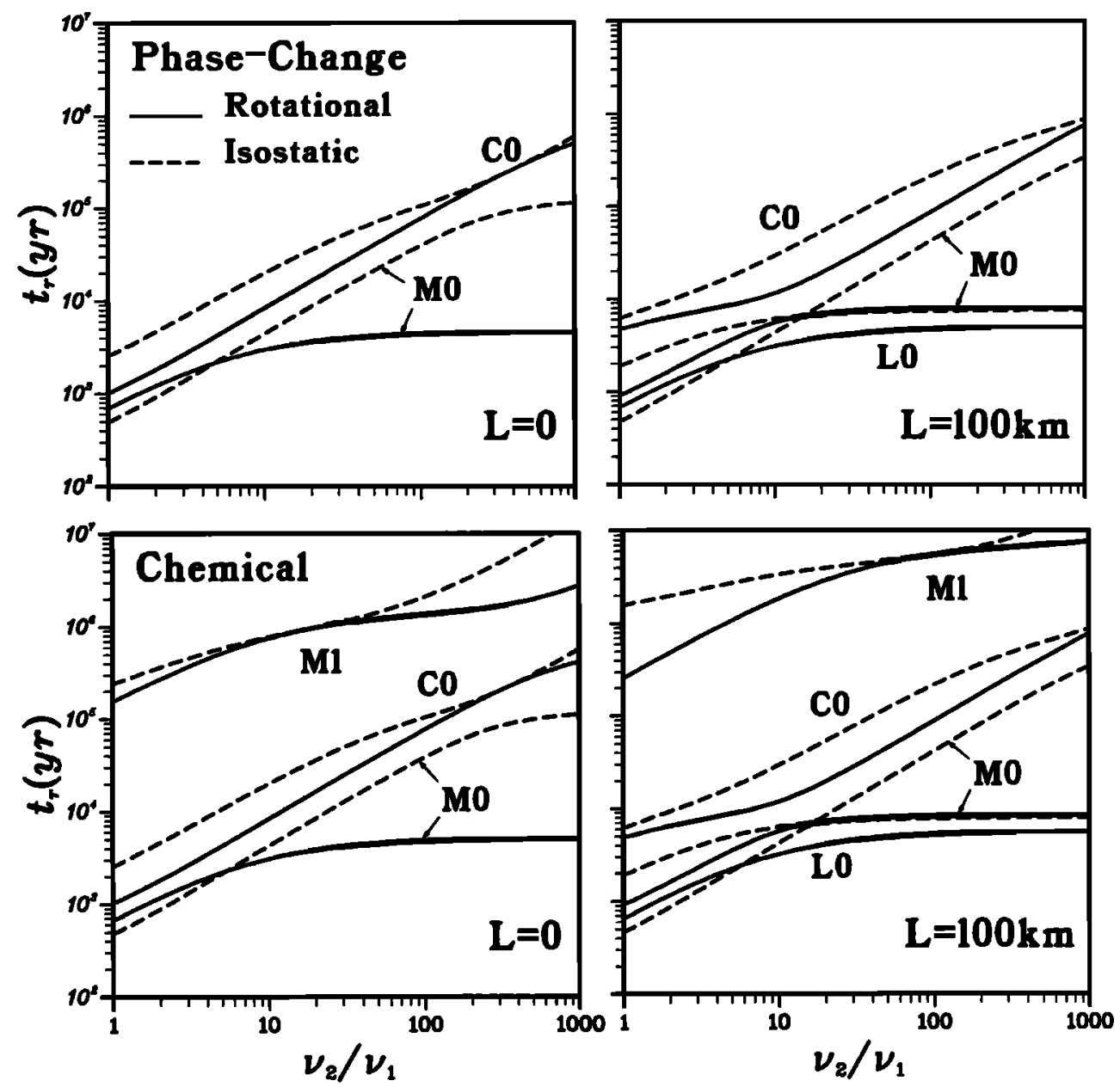

Fig. 6. Characteristic isostatic (dashed lines) and rotational (solid lines) times as a function of the ratio between lower and upper mantle viscosit.jes. The upper-lower mantle interface is either an adiabatic phase change (top) or a chemical boundary (bottom). The effect of the lithospheric mode is only present in the two right panels. The slower mode is always an isostatic mode.

ferent depths, and eight Earth models are considered. The displacements in degrees are computed as a function of time expressed in millions of years after the loading occurring at time $t=0$. By convention, the pole displacement is positive when the axis of rotation is wandering toward the source, negative when it is wandering away from the source. In other words, described in a reference frame where the rotation axis is fixed, the positive mass anomaly introduced at $45^{\circ}$ drifts toward the pole when $m$ is positive and drifts toward the equator when $m$ is negative.

Three features are immediately noticeable. First, very large polar shifts or drifts can be achieved. Second, different viscosity stratifications or boundary conditions at 670 km lead to opposite directions of polar wander. Third, a mass located close to a phase change interface acts very differently in comparison with a mass located close to a chemical interface. For the phase change case in the four top panels, a polar drift at constant velocity is excited. The two top panels (models a and b) corresponding to uniform mantle viscosity of $10^{21} \mathrm{~Pa} s$ show unrealistically large displacements, much larger than allowed by linearization. For displacements larger than $10^{\circ}$, the dashed lines remind the reader that in this case the solutions are no more consistent with the linearized approximation used here. A viscosity increase by a factor of 30 (models $c$ and d) slows down the polar motion by more than 1 order of magnitude. In the four bottom panels, the major effect of chemical stratification is a further huge reduction in the polar excursions. In this case, the polar wander velocity for sources close to the 670-km boundary tends to zero after a few million years, and a polar shift of finite amplitude is reached.

These results emphasize the deficiency of rigid Earth models in the interpretation of true polar wander data induced by internal sources as subductions, hotspots, or mantle insulation due to continental aggregation [Anderson, 1982; Ju$r d y, 1983]$. They suggest that polar wander data can be used to put bounds on the amount of chemical and viscosity stratification in the mantle.

The qualitative difference between polar wander induced by surface and by internal loadings is easy to understand. After relaxation, the excitation due to surface loading tends to zero except for a very low nonisostatic contribution frozen by the elastic lithosphere. Therefore the polar wander velocity vanishes after a few tens of thousands of years. After a similar relaxation time, a surface deflection roughly compensates an internal mass. However, the deep mass and 

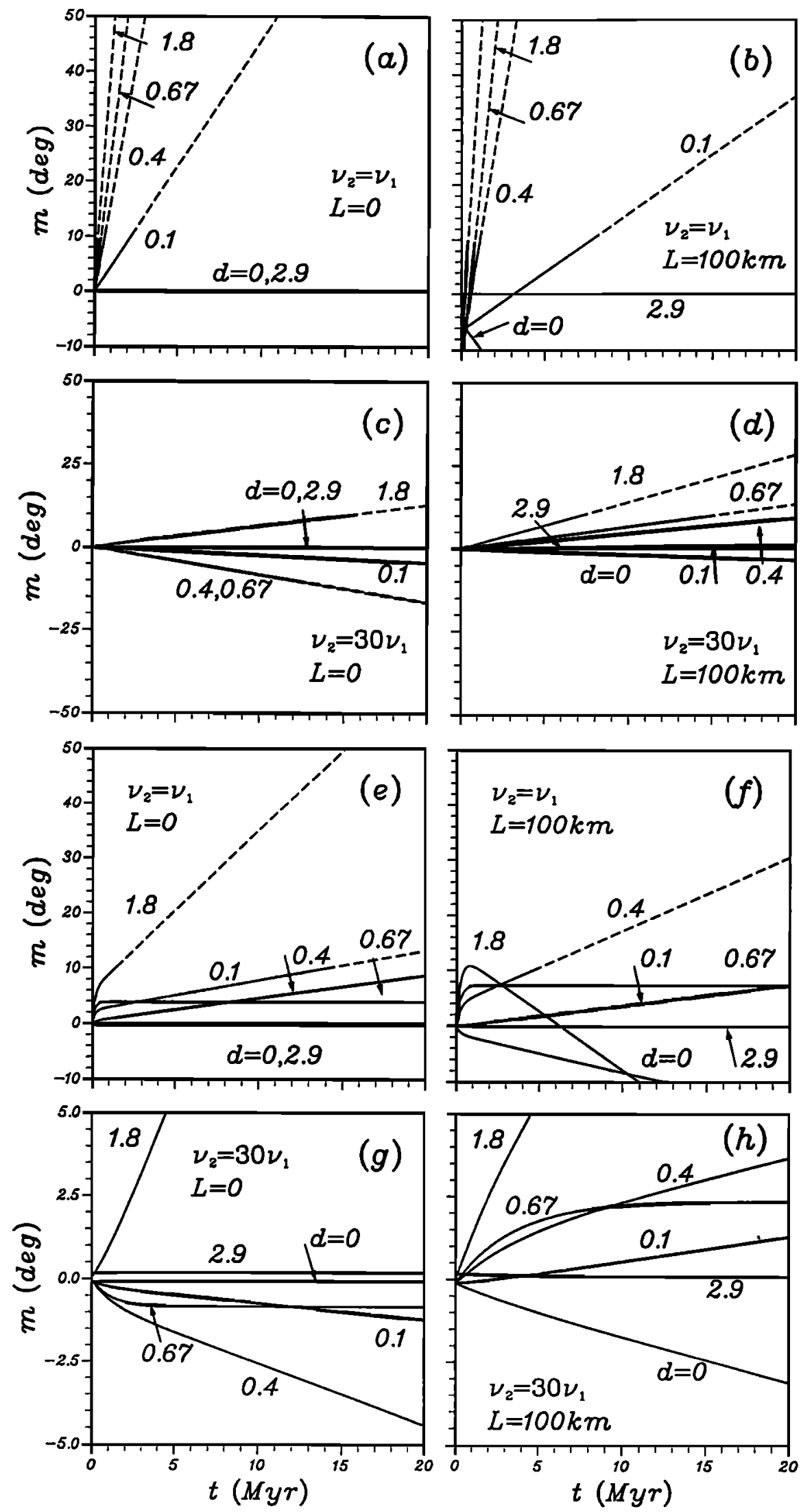

Fig. 7. Displacement of the axis of rotation induced by a mass anomaly located in the mantle at different depths given in units of $10^{3} \mathrm{~km}$. This mass anomaly simulating a slab is seated at $45^{\circ}$ from the pole and acts at $t=0$. The displacement is positive when the mantle rolls in such a way that the mass anomaly is moved toward the pole. The eight panels correspond to the eight viscosity stratifications presented in Table 1. According to the linearization our solutions are only valid for small pole displacements, say, lower than $10^{\circ}$. Notice that the vertical scale is different in the various panels, and the smallest displacements are obtained for a viscosity increase in the lower mantle (models $\mathrm{c}, \mathrm{d}, \mathrm{g}$, and $\mathrm{h}$ ). 
the surface deflection being at different distances from the Earth's center, they lead to a finite contribution to the nondiagonal element of the inertial tensor. In that case, the polar wander reaches a constant value different from zero except for mass anomalies located close to a chemical interface. If we neglect the redistribution of masses due to convection, the polar wander excitation driven by internal masses can only disappear after the reorientation of the rotational axes in a new position where the two off-diagonal terms $I_{x z}$ and $I_{y z}$ are zero.

The Earth is always wandering toward a configuration where the main inertial momentum that would have existed in a nonrotating Earth coincides with the rotation axis. Let us take the example of a positive mass anomaly introduced in the mantle. Just after the initial elastic shot, the internal density anomaly remains strongly undercompensated, and the Earth tends to bring this anomaly to the equator. This occurs on such a short time span that it is difficult to see with a linear time scale. However, it explains why in the model b panel of Figure 7 , the displacement curves seem to start from negative values. Then, the surface is relaxing viscously, and its effect on the inertial tensor can overcome the effect of the deep-seated mass. In that case, the nondiagonal inertial terms change signs, and the rotation axis drifts toward the internal anomaly. The long-term behavior of polar wander, toward or away from the internal mass, is controlled by the steady state Green's functions relating the internal mass to the geoid. The existence in the mantle of a chemical stratification imposes a zero long-term geoid and zero off-diagonal inertial products for masses close to such an interface. This leads to a finite polar shift reached after a time controlled by the slow M1 mode that enters in both the isostatic and the rotational part of the Liouville equation (3).

In the previous computations the slabs were supposed to act instantaneously. In the following simulations, we get rid of this unrealistic hypothesis. A slab of lateral extension $4000 \mathrm{~km}$, located at $45^{\circ}$, is vertically sinking in the mantle with a constant velocity $v=2,5$, or $10 \mathrm{~cm} / \mathrm{yr}$. At each time corresponding to a given downward movement, a new mass anomaly is introduced ahead of the slab and starts to act. The total mass introduced in the mantle is $2 . \times 10^{19} \mathrm{~kg}$ when the stem of the slab reaches $670-\mathrm{km}$ depth. We only run our computations for models without lithosphere.

Figure 8 depicts our results for a phase change stratification (top) and a chemical one (bottom). Because of our more realistic mass redistribution history, the polar wander velocity is zero when the subduction process begins. The slab having a velocity of $10 \mathrm{~cm} / \mathrm{yr}$ reaches the upper-lower mantle interface in $6.7 \mathrm{~m} . \mathrm{y}$.. In this case we assume that the subduction process stops but the mass anomaly still continues to influence the isostatic and rotational processes. A
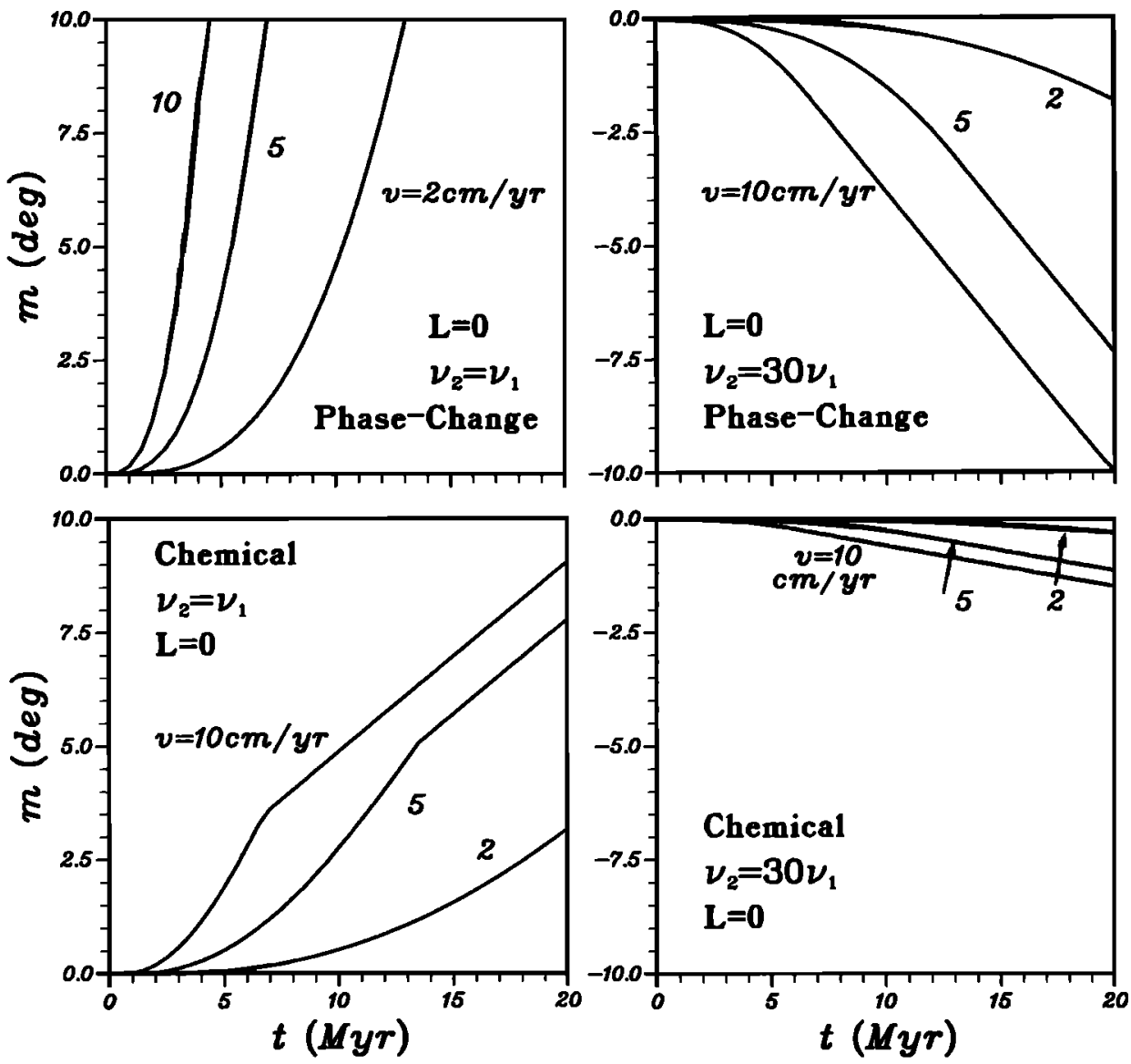

Fig. 8. Displacement of the axis of rotalion induced by a slals diving in the mantle with velocity 2,5, or $10 \mathrm{~cm} / \mathrm{yr}$. At time $t=0$ the subduction initiates at $45^{\circ}$ from the north pole. The upper-lower mantle interface is a phase change on top, a chemical boundary on bottom. The mantle viscosity is uniform in the left column and increases by a factor of 30 in the right column. No lithosphere is included. 
kink in the curves related to this end of subduction is noticeable in the bottom left panel. Before the slab reaches the depth of the upper-lower mantle interface, its mass anomaly is lower than the mass anomaly used in Figure 7. The polar displacement values are much more realistic than what was found in our drastic previous computations. However, the qualitative characteristic features of Figure 7 are the same. First, a viscosity increase and furthermore a chemical stratification drastically decrease the amount of polar wander. Second, the slab moved toward the equator in the isoviscous case and toward the pole when the viscosity is increasing at depth.

\section{CONCLUDING REMARKS}

We have proven that changes in the upper mantle density distributions are sizeable contributors to polar wander. On the basis of our results, we can conclude that any attempt to estimate the moment of inertia or polar wander due to internal density distribution within the framework of a rigid Earth model is incorrect. A realistic mantle acts to compensate the anomalous densities. The induced topographies of the displaced boundaries, like the Earth's surface, the $670-\mathrm{km}$ discontinuity, and the core-lower mantle interface often overcome the direct effects of the source. This dynamic process is very sensitive to the boundary conditions prevailing on the top of the planet, free slip or no slip, and to the presence of viscosity and/or chemical stratification in the mantle. A physically stratified mantle allows much more rapid polar wander than a chemical layered one. A same source induces opposite polar wander directions, for different mechanical stratifications. The depth of the internal source, both for phase change and chemically stratified models, is a crucial parameter that controls the amount of polar wander. For a chemical upper-lower mantle interface and a source embedded in proximity of this transition, a long-term polar wander is forbidden, owing to complete isostatic compensation. The same is true at the core-mantle interface.

A comparison can be drawn between the results that may be obtained from the present-day geoid and from the TPW. The two subjects are closely related. However, the polar wander modelization seems to be more sensitive to the internal mass anomalies because the mantle dynamics enters two times in the equations: through the isostatic modes and through the rotational modes.

The next step of our work will be to try to model the TPW detected from paleomagnetism using our model. We already suspect that the present-day TPW is mainly related to postglacial rebound rather than to mantle mass redistribution. We also know that the present-day internal mass anomalies revealed by seismic tomography may drive a very slow polar wander [Ricard and Sabadini, 1990]. The understanding of TPW will come from the history of mantle circulation. This history will remain out of reach, but the time evolution of the subduction zones in the past $200 \mathrm{~m} . \mathrm{y}$. may be hypothesized as forcing the basic features of mantle circulation [Bunge and Richards, 1990; Richards and Engebretson, 1992]. If this hypothesis holds, we can hope to put constraints on the mantle structure by modeling the TPW.

The interpretation of the real TPW is beyond the scope of this paper. However, some preliminary conclusion can be drawn. An observed TPW of about $10^{\circ}$ seems to have occurred in the past $40 \mathrm{~m} . \mathrm{y}$. in the direction of Greenland
[Besse and Courtillot, 1991]. Between 110 and 40 m.y. a polar wander of $20^{\circ}$ took place in the opposite direction. The implied low polar velocity of around $0.3^{\circ} / \mathrm{m} . \mathrm{y}$. suggests that a uniform mantle with viscosity of $10^{21} \mathrm{~Pa} s$ cannot explain the data and that a large mantle stratification is needed in order to stabilize the Earth's rotational axes. The fact that in the last tens of millions of years the main subduction zones were situated in the western Pacific area at a longitude roughly opposite Greenland toward which is wandering the Earth also strengthens the necessity of a mantle viscosity increase: only in that case, dense mass anomalies can be brought to the pole.

Acknowledgments. We are grateful to David A. Yuen and Jacques Hinderer for helpful comments. This work was partly supported by the INSU-DBT (Dynamique et Bilan de la Terre) program (Global Dynamics, contribution 430) and by the SCIENCE program of the European Economical Community.

\section{REFERENCES}

Anderson, D. L., Hotspots, polar wander, Mesozoic convection and the geoid, Nature, 297, 391-393, 1982.

Besse, J., and V. Courtillot, Revised and synthetic apparent polar wander paths of the African, Eurasian, North American and India plates, and true polar wander since $200 \mathrm{ma}, J$. Geophys. Res., 96, 4029-4050, 1991.

Bunge, H. P., and M. A. Richards, Modelling the relationship between subduction and large-scale mantle heterogeneity: The effects of depth-dependent viscosity, (abstract), EOS Trans. AGU, 71, 1625, 1990.

Cathles, L. M., The Viscosity of the Earth's Mantle, Princeton University Press, Princeton, N. J., 1975.

Courtillot, V., and J. Besse, Magnetic field reversals, polar wander, and core-mantle coupling, Science, 237, 11401147, 1987.

Dickman, S. R., Investigation of controversial polar motion features using homogeneous international latitude service data, J. Geophys. Res., 86, 4904-4912, 1981.

Duncan, R. A., and M. A. Richards, Hotspots, mantle plumes, flood basalts, and true polar wander, Rev. Geophys., 29, 31-50, 1991.

Fleitout, L., and C. Moriceau, Topography and geoid anomalies due to density heterogeneities at the base of the thermal lithosphere: Application to oceanic swells and small wavelength geoid lineations, Geophys. J. Int., 107, 265-277, 1991.

Forte, A. M., and W. R. Peltier, Mantle convection and aspherical Earth structure: The importance of poloidaltoroidal coupling, J. Geophys. Res., 92, 3645-3679, 1987.

Goldreich, P., and A. Toomre, Some remarks on polar wandering, J. Geophys. Res., 7ł, 2555-2567, 1969.

Hager, B. H., R. W. Clayton, M. A. Richards, R. P. Comer, and A. M. Dziewonski, Lower mantle heterogeneity, dynamic topography, and the geoid, Nature, 313, 541-545, 1985.

Hargraves, R. B., and R. A. Duncan, Does the mantle roll?, Nature, 245, 361-363, 1973.

Haskell, N. A., The motion of a viscous fluid under a surface load, Physics, 6, 265-269, 1935.

Hinderer, J., H. Legros, and M. Amalvict, A search of Chandler and nearly diurnal free wobbles using Liouville equa- 
tions, Geophys. J. R. Astron. Soc., $\gamma_{1}, 303-322,1982$.

Hinderer, J., D. Jault, H. Legros, and J. L. LeMouel, Core mantle topographic torque: A spherical harmonic approach and implications for excitation of the Earth rotation by core motions, Phys. Earth Planet. Inter., 59, 329-341, 1990.

Hinderer, J., H. Legros, and D. Crossley, Global Earth dynamics and induced gravity changes, J. Geophys. Res., $96,20,257-20,265,1991$.

Jurdy, D. M., Early Tertiary subduction zones and hotspots, J. Geophys. Res., 88, 6395-6402, 1983.

Kaula, W. M., Elastic models of the mantle corresponding to variations in the external gravity field, J. Geophys. Res., 68, 4967-4978, 1963.

Lambeck, K., Geophysical Geodesy, Oxford University Press, New York, 1988.

Lefttz, M., and H. Legros, Some remarks about the rotations of a viscous planet and its homogeneous liquid core: Linear theory, Geophys. J. Int., 108, 705-724, 1992.

Morgan, W. J., Convection plumes in the lower mantle, $\mathrm{Na}$ ture, 230, 42-43, 1971.

Munk, W. H., and G. J. F. MacDonald, The Rotation of the Earth, Cambridge University Press, New York, 1960.

Nakiboglu, S. M., and K. Lambeck, Deglaciation effects on the rotation of the Earth, Geophys. J. R. Astron. Soc., 62, 49-58, 1980.

Ockenden, J. R., and D. L. Turcotte, On the gravitational potential and field anomalies due to thin mass layers, Geophys. J., 48, 479-492, 1977.

Ricard, Y., and R. Sabadini, Rotational instabilities of the Earth induced by mantle density anomalies, Geophys. Res. Lett., 17, 627-630, 1990.

Ricard, Y., and C. Vigny, Mantle dynamics with induced plate tectonics, J. Geophys. Res., 94, 17,543-17,559, 1989.

Ricard, Y., L. Fleitout, and C. Froidevaux, Geoid heights and lithospheric stresses for a dynamic Earth, Ann. Geophys., 2, 267-286, 1984.

Richards, M. A., and D. C. Engebretson, Large-scale mantle convection and the history of subduction, Nature, 355, 437-440, 1992.

Richards, M. A., and B. H. Hager, Geoid anomaly in a dynamic Earth, J. Geophys. Res., 89, 5987-6002, 1984.

Sabadini, R., and W. R. Peltier, Pleistocene deglaciation and the Earth's rotation: Implications for mantle viscosity, Geophys. J. R. Astron. Soc., 66, 553-578, 1981.

Sabadini, R., and D. A. Yuen, Mantle stratification and long-term polar wander, Nature, 339, 373-375, 1989.

Sabadini, R., C. Doglioni, and D. A. Yuen, Eustatic sea level fluctuations induced by polar wander, Nature, 345, 647-650, 1990.
Sabadini, R., D. A. Yuen, and E. Boschi, Polar wander and the forced responses of a rotating, multilayered, viscoelastic planet, J. Geophys. Res., 87, 2885-2903, 1982.

Sabadini, R., D. A. Yuen, and E. Boschi, A comparison of the complete and truncated versions of the polar wander equations, J. Geophys. Res., 89, 7609-7620, 1984.

Sabadini, R., D. A. Yuen, and P. Gasperini, The effects of transient rheology on the interpretation of lower mantle viscosity, Geophys. Res. Lett., 12, 361-364, 1985.

Spada, G., R. Sabadini, D. A. Yuen, and Y. Ricard, Effects on post-glacial rebound from the hard rheology in the transition zone, Geophys. J. Int., 109., 683-700, 1992.

Spada, G., D. A. Yuen, R. Sabadini, P. J. Morin, and P. Gasperini, A computer-aided, algebraic approach to the post-glacial rebound problem, Math. J., 1, 65-69, 1990.

Takeuchi, H., M. Saito, and N. Kobayashi, Statical deformations and free oscillations of a model Earth, J. Geophys. Res., 67, 1141-1154, 1962.

Tanimoto, T., Moment of inertia of three-dimensional models of the Earth, Geophys. Res. Lett., 16, 389-392, 1989.

Turcotte, D. L., and G. Shubert, Geodynamics, John Wiley, New York, 1982.

Wilson, C. R., and R. A. Vicente, Estimates of the Chandler's component of polar motion as derived from various data sets, Astron. Nachr., 302, 226-232, 1981.

Wu, P., Deformation of internal boundaries in a viscoelastic Earth and topographic coupling between the mantle and core, Geophys. J. Int., 101, 213-231, 1990.

Wu, P., and W. R. Peltier, Glacial isostatic adjustment and the free air gravity anomaly as a constraint of deep mantle viscosity, Geophys. J. R. Astron. Soc., 74, 377-449, 1982.

Wu, P., and W. R. Peltier, Pleistocene glaciation and the Earth's rotation: A new analysis, Geophys. J. R. Astron. Soc., 76, 753-791, 1984.

Yuen, D. A., and L. Fleitout, Thinning of the lithosphere by small-scale convective destabilization, Nature, 319, 125$128,1985$.

Yuen, D. A., R. Sabadini, and E. Boschi, The viscosity of the lower mantle as inferred from rotational data, J. Geophys. Res., 87, 10,745-10,762, 1982.

Y. Ricard, Département de Géologie, Ecole Normale Supérieure, 24, rue Lhornond, 75231 Paris, Cedex 05, France.

R. Sabadini and G. Spada, Dipartimento di Fisica, Settore di Geofisica, Università di Bologna, Viale Berti Pichat 8, 40127 Bologna, Italy.

(Received September 18, 1991; revised March 4, 1992; accepted March 14, 1992.) 